ISSN 1112-9867

Available online at

http://www.jfas.info

\title{
A STUDY ON THE PERCENT OF FREQUENCY OF ACME-ARCA IN CLINICAL ISOLATES RESISTANT TO METHICILLIN- STAPHYLOCOCCUS EPIDERMIDIS IN ISFAHAN, IRAN
}

\author{
S. Shamansouri ${ }^{1}$, V. K. Zadeh ${ }^{2}$, M. Khazaei ${ }^{3}$ \\ ${ }^{1}$ Microbiology researcher, Falavarjan Branch, Islamic Azad University, Isfahan, Iran \\ ${ }^{2}$ Assistant Professor in Medical Microbiology,Falavarjan Branch, Islamic Azad University, \\ Isfahan, Iran \\ ${ }^{3}$ Assistant Professor of Plant Biotechnology, College of Biology, IsfahanUniversity, Isfahan, Iran
}

Published online: 19 June 2016

\begin{abstract}
ACME is a mobile element of Arginine catabolic in Staphylococcus epidermidis that codes specific virulence factors. The purpose of this study was to examine the specific features and prevalence of ACME-arcA in the isolates of Staphylococcus epidermidis resistant to Methicillin isolated by clinical samples in Isfahan. In a time interval of 9 months, 120 clinical samples were gathered through Asgariyeh Hospital. After identification of the isolates, their antibiotic susceptibility was determined by disk diffusion method. Resistance to cefoxitin was measured using agar screening method and MIC values was measured using Espilometer Test (E Test). $M e c A$ and ACME-arcA genes were detected by PCR technique. Out of 120 clinical samples, 68 Staphylococcus epidermidis strains were isolated. Most isolates were obtained from urine samples. In this study, 51 isolates $(75 \%)$ were resistant to cefoxitin and multiple drug resistance was observed in 50 isolates $(73.53 \%)$.
\end{abstract}

Author Correspondence, e-mail: sh.shamansouri@yahoo.com

doi: http://dx.doi.org/10.4314/jfas.v8i2S.119 
Studies show that this is the first study on gene detection of ACME-arcA in isolates of Staphylococcus epidermidis in Iran. It was found that Staphylococcus epidermidis with ACMEarcA reservoirs is highly correlated to virulence and antimicrobial resistance genes.

Keywords: PCR, Etest, mecA, ACME-arcA.

\section{INTRODUCTION}

Coagulase negative staphylococci is an important part of normal microbial flora of the skin and mucosa. Coagulase negative staphylococci prefer specific niches and can be colonized anywhere on the body. Clonization plays important role in organism survival, so that changes it to an important pathogensand results a biofilm-related infections in the Medical restoration when penetrating the epithelial barriers (Lindgren et al, 2014 Du X et al, 2013)

Molecular factors involved in the success of Staphylococcus epidermidis in the colonization of pathogenic involves genes and proteins that facilitate the spread of infection and the organism in the body and plays an important role in the Commensalism life of this organism (Lindgren et al, 2014; Rosenthal et al , 2011).

Staphylococcus epidermidis produces biofilm that is a dense and viscous mass of bacteria in the extracellular matrix. Biofilm activities include antibacterial resistance, resistance to antibiotics and increase resistance to immune response (decreased oxygen and nutrients tilt) (Shore et al, 2011; Thurlow et al, 2013).

Staphylococcus epidermidis biofilm is a complex mixture of extracellular DNA (eDNA), intracellular polysaccharide (PIA), mass binding protein (Aap) and protein binds to the extracellular matrix (Embp) that plays important role in forming the biofilms and connect it to fibronectin and cell. In addition, it includes Bap / Bhp adhesions that play important role in mass density and also protects poly-gamma-glutamic acid (PGA) of bacteria against neutrophils killer. (Otto, 2011).

Arginine catabolic mobile element (ACME) is a new genomic island to increase the capacity of colonization of Staphylococcus species in the skin and mucus and plays an important role in survival and virulence of the organism (Borui et al, 2006).

ACME has gene cluster of opp-3 and arc that are homologous each other and involved in bacterial species in the transmission of the disease. Arc Gene (arcA, $\operatorname{arcB}, \operatorname{arc}, \operatorname{arcD})$ is 
composed of six gene cluster that encodes the D-arginine deaminase route that convert Larginine to carbon dioxide, ATP and ammonium to carbon dioxide and this route is important in survive in low $\mathrm{pH}$ and to prevent the host immune response against bacterial infection (Degnan et al, 2000; Moncada And Higgs, 1993).

D-arginine deaminase route is a main route in bacteria that arginine is the only source of energy for their growth. It should be noted that the arc gene islocated in Staphylococcus and Pseudomonas aeruginosa isolates on chromosome. Three genes of $\operatorname{arcA} \operatorname{arcC}$, $\operatorname{arcB}$, respectively, are located on the arc apron which causes somecatalytic change of arginine to Ornithine, arginine to ammonia and arginine to carbon dioxide and ATP also is produced, when they are producing. Arginine catabolic mobile element is a DNA genetic region that is integrated into the OrfX. This element binds to SCCmec element in methicillin-resistant Staphylococcus and increases virulence and colonization ability of Staphylococcus in humans, but so far the origin of the arrival of the elements toSCCmec has not been recognized (Barbier et al, 2011; Diep et al, 2008; Henegariu et al, 1997).

ACME is in the lower area of Cassette chromosome of mec Staphylococcal and make ACMESCCmec combination together. Physical connection between SCCmec and ACME points out that the selection of antibiotic resistance and pathogenicity may be interconnected (Urushibara et al, 2012).

Opp-3 cluster is a member of the ABC Transporters family that bind to upstream peptides nutrients, Quorum sensing, pheromone transport, chemiotaxis, adhesions of eukaryotic cells, binding protein composition and expression of virulence determinants. This gene encodes anOligopeptide permease(Podbielski et al, 1996). There is a hypothesis that reducing the host inflammatory response is indirectly associated with deficiency in L - arginine and inhibition of cell responses nuclease menu and this is a subsidiary of nitric oxide production in microorganisms. Previous studies have shown that high levels of ACME among strains of Staphylococcus epidermidis is a major advantage granted to other species (Elington et al, 2008). (Acme-arcA) Compounds determines virulence and resistance factors and dedicated to clinical sites that allow attachment of the influx and proliferation in the organism.

Otherwise, the organism is commensalism. Staphylococcus epidermidis is normally very little invasive or may not be completely safe, reports have shown that the molecular characterization 
of Staphylococcus epidermidis with the acquisition of mobile genetic elements can be in a perfect position to produce biofilm infections in restoration or even in the absence of a steady increase biofilm bacteria virulence, for the creation of antimicrobial resistance and tolerance to the drug's safety (Bartels et al, 2011; Miragaia et al, 2009). The aim of this study was to evaluate ACME gene in isolates of Staphylococcus epidermidis isolates from clinical hospitals in Isfahan.

\section{METHODOLOGY OF STUDY}

In this descriptive study that was conducted from May to December 2014, 120clinical samples were collected from inpatient and outpatient in Askariye hospital and Nobel medical diagnostic laboratory of Isfahan. Samples include urine, blood, spinal fluid, vaginal, wound, throat and sputum chips. Out of 120 isolates, $22(18.33 \%)$ of isolates belonged to outpatients and 98 $(81.66 \%)$ of isolates were belonged to hospitalized patients. After the initial separation, isolates were identified using conventional biochemical methods including catalase test, coagulase tube, mannitol salt agar, novobiocin and nucleases deoxyribose (all Manufacturing by Himedia company of India) (Quinn et al, 1994).

Antibiotic resistance patterns of isolates to oxacillin, gentamicin, cefoxitin and rifampin (purchased from the Himedia Company of India) were determined using Kirby-Bauer method based on CLSI protocol (Borui et al, 2009; Du X et al, 2013).

For primary isolation of the isolates resistant to methicillin and cefoxitin, agar screening method was used. In short, the Mueller plates of Hinton agar containing $6 \mu \mathrm{g} / \mathrm{ml}$ from antibiotic cefoxitin was prepared. Then, from 24 hour culture of the suspension bacterial, half was Sample of McFarland was prepared and $110 \mu \mathrm{l}$ of suspensionwas inoculated on a point of medium using a sampler. Plates were incubated for 24 hours in $37^{\circ} \mathrm{c}$. After this time, the growth of the isolates were analyzed according to CLSI protocol (Palazzo et al, 2005; Shama et al, 2010).

To determine the antibiotic cefoxitin MIC, Espilometer (E Test method) was used. To do test, a suspension equivalent to half McFarland was prepared in 24-hour culture tests for bacteria Mueller-Hinton agar. Then, the bars were placed on the environment, and the plates were incubated for 24 hours in $37 \circ \mathrm{C}$. Then, the MIC was read from the bar in accordance with CLSI protocols. Cefoxitin with MIC greater or equal to $24 \mathrm{ml} / \mathrm{g}$ was considered for resistant strain of less than or equal and8g / $\mathrm{ml}$ was sensitive strain (Palazzo et al, 2005). 
DNA was extracted by boiling method. identification of mecA and ArcA gene in isolated Staphylococcus epidermidis strains were done using thermal cycle specific primers for these genes (Table 1,2,3) 11 of DNA extracted and 11 of each primer was added to PCR Master kit (Kappa Country USA) with the final volume of 25 1. S. aureus ATCC 33591 and ATCC 25923 in the whole process were used as positive and negative controls. bp 100 marker was used to verify the molecular weight. Electrophoresis of samples on $1 \%$ agarose gel were stained with Midory viewer and the results was observed with Gel doc (Bio-RAD) UV light (Kondo et al, 2007; Onishi et al, 2013).

\section{FINDINGS}

From May to December 201 ,68 isolates of Staphylococcus epidermidis were collected from Asgariye hospital and Nobel medical laboratory. A total of 12 isolates (17.6\%) belonged to outpatients and 56 isolates $(52.35 \%)$ belonged to hospitalized patients.

Table 4 shows isolates in clinical sample separation. According to Kirby Bauer, 40 isolates (58.82\%) were resistant to cefoxitin (Table 5). According to Agar screening results, 39 isolates (55.88\%) was resistant to cefoxitin. With E Test, 39 isolates (55.88\%) were resistant to cefoxitin. Using PCR showed that 39 isolates resistant to cefoxitin, 22 isolates (56.41\%) had mecA gene, 15 isolates (38.46\%) had arcA gene. Of these, 14 isolates (20.58\%) also had both mecA gene and gene $\operatorname{arcA}$ (Figure 1 and 2).

Out of isolates, five isolates were false positive, 2 were false negative of mecA and 3 were false positive and one were false arcA negative samples.

\section{DISCUSSION AND CONCLUSION}

According to studies, two major factors are important in the pathogen of Staphylococcus epidermidis in infections. Acquired antibiotic resistance genes are the first factor and the second factor is that the bacteria produce biofilms is out of the reach of antibiotics. A patient who is 
admitted to the hospital is colonized very soon using Staphylococcus epidermidis biofilm formation in the environment or medical personnel (Ellington et al, 2008; Sabat et al, 2015).

In Akhtardanesh et al at Arak on ICU patients, the rate of methicillin resistance in S. aureus was 77.4\% and all samples were positive in ACME (Akhtardanesh et al., 2015). Ellington et al in a study in 2010 in England founded 13 positive isolates of ACME out of 17 isolates of Staphylococcus epidermidis. In a study by Shoor et al, 238 strains of S. aureus were isolated of Ireland, 23 isolates $(9.7 \%)$ had positive ACME.

ACME-arcA is a genomic island and a protected sequence in Staphylococcus that plays important role in survival and virulenceand is emerged in many acquired strains of community and hospital (Kawaquchiya et al, 2013).

Studies have shown that ACME-arcA in Staphylococcus aureus virulence may directly have no effect on virulence factors, but is important in growth, survival, and colonization of host (Kawaquchiya et al, 2012).

Brown et al in a study isolated 1055 strains of Staphylococcus aureus strains and $77.4 \%$ had MRSA strains and 3.54\% of MSSA strains were positive in terms of ACME gene. In a study by Deep et al in 2008, the prevalence of ACME gene was reported in $60 \%$ of Staphylococcus aureus isolates andall were MRSA.

Granslou et al reported in 2010, $23 \%$ of isolates of Staphylococcus epidermidis isolated from blood cultures $40 \%$ were Staphylococcus epidermidis strains isolated from blood cultures infected with the ACME gene. In addition, all isolates showed resistance to antibiotics. In research by Marzhyna et al in 2009, 51\% of Staphylococcus epidermidis expanded in geographical regions were ACME positive.

In the study of Gozin Do et al in 2013 in a hospital in Shanghai, the distribution of ACME in Staphylococcus epidermidis was $54.8 \%$ and was shown that ACME genes can lead to the growth, survival and spread of multidrug-resistant strains.

A recent study of 68 isolates of Staphylococcus epidermidis, 22 isolates (56.41\%) were methicillin-resistant and 15 isolates (46/38\%) were ACME positive and 14 (58/20) isolates had both genes. The obtained results were fewer than other studies. We assume that the difference in the geographic region can be caused by genetic variation and deviation or emerging this gene slowly in this region. Totally, the results of study showed a significant relationship between 
medical resistance and ACME gene. So, sufficient attention should be paid to use Antibiotics. Also, genotype experiments can be used instead of phonotype experiments to facilitate the procedure and treatment. Observing Hygiene principles and periodic control of personnel will also prevent the spread of disease.

\section{Appreciation}

Thereby, we appreciate Dr. Mahdi Khazaei, Mr. Amir Jafarpour and personal of bacteriology sector of Asgariye Hospital of Isfahan as well as Dr. Ajami and Ms. Zohreh Nasab and Ms. Mosavi.

Table 1: Thermal Cycle Used To Identify The Meca Gene.

\begin{tabular}{|c|c|c|}
\hline PCR Steps & temperature & Time \\
\hline Initial denaturation & 96 & $5 \mathrm{~min}$ \\
\hline Denaturation & 94 & $45 \mathrm{~min}$ \\
\hline Annealing & 45.5 & $60 \mathrm{~min}$ \\
\hline Extension & 72 & $1 \mathrm{~min}$ \\
\hline Final extension & 72 & $10 \mathrm{~min}$ \\
\hline Final extension & 35 & \\
\hline
\end{tabular}

Table 2: Thermal Cycle Used To Identify theACME-Arca Gene

\begin{tabular}{|c|c|c|}
\hline PCR Steps & temperature & Time \\
\hline Initial denaturation & 96 & $5 \mathrm{~min}$ \\
\hline Denaturation & 94 & $45 \mathrm{~min}$ \\
\hline Annealing & 45.5 & $60 \mathrm{~min}$ \\
\hline Extension & 72 & $1 \mathrm{~min}$ \\
\hline Final extension & 72 & $10 \mathrm{~min}$ \\
\hline Final extension & 35 & \\
\hline
\end{tabular}

Table 3: Primers used in the PCR

\begin{tabular}{|c|c|c|}
\hline Gene & Primer & Size of bp Product \\
\hline mecA & F: TGC TAT CCA CCC TCA AAC AGG & 286 \\
& R: AAC GTT GTA ACC ACC CCA AGA & \\
\hline arcA & F: GAG CCA GAA GTA CGC GAG & 486 \\
& R: CAC GTA ACT TGC TAG AAC GA & \\
\hline
\end{tabular}


Table 4- The Frequency of Staphylococcus epidermidis strains isolated by Clinical Samples

\begin{tabular}{|c|c|c|}
\hline Kind of Infection & Number of Sample & S. epidermidis Isolate \\
\hline Urine & 92 & $83(77.94 \%)$ \\
\hline Blood & 14 & $7(10.29 \%)$ \\
\hline Vaginal & 5 & $3(4.41 \%)$ \\
\hline Wound & 4 & $2(2.94 \%)$ \\
\hline Spinal fluid & 1 & $1(1.74 \%)$ \\
\hline Tracheal Intubation & 1 & $1(1.74 \%)$ \\
\hline Throat & 1 & $1(1.74 \%)$ \\
\hline Sputum & 2 & - \\
\hline Total & 120 & 68 \\
\hline
\end{tabular}

Table 5: Antimicrobial Susceptibility of Strains S.Epidermidis

\begin{tabular}{|c|c|c|c|}
\hline Antibiotic & Sensitive & Semi-sensitive & Resistant \\
\hline Cefoxitin & $17(25 \%)$ & $9(13.23 \%)$ & $41(60.29 \%)$ \\
\hline oxacillin & $9(13.23 \%)$ & - & $54(74.41 \%)$ \\
\hline Gentamicin & $39(57.35 \%)$ & $16(23.52 \%)$ & $22(32.35 \%)$ \\
\hline Rifampin & $18(26.47 \%)$ & $48(70.58 \%)$ & $25(36.76 \%)$ \\
\hline
\end{tabular}

\section{$\begin{array}{llllllllllll}1 & 2 & 3 & 4 & 5 & 6 & 7 & 8 & 9 & 10 & 11 & 12\end{array}$}

bp 286

GenemecA

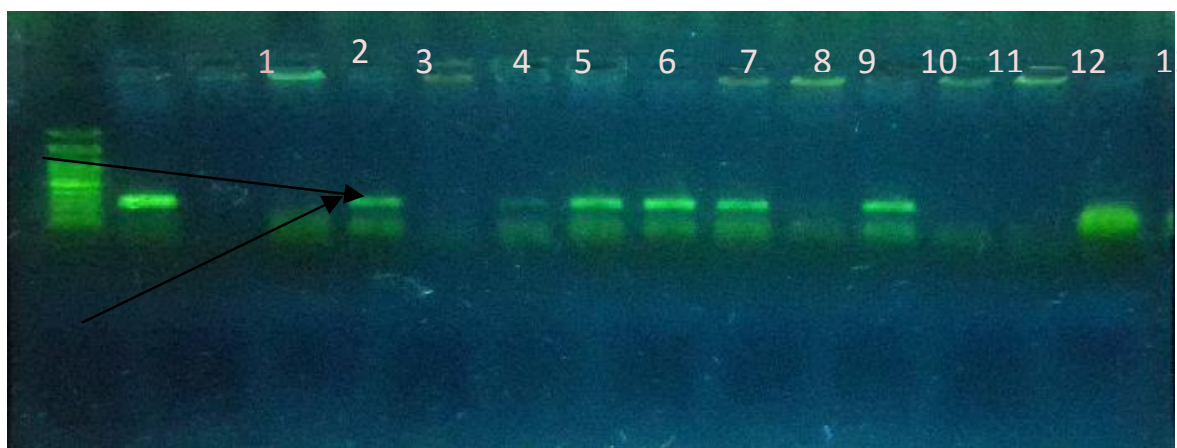


Fig.1. PCR reaction in mecA gene in isolates of MRSE: Well 1: bp 100 molecular weight marker, wells 2: positive control of ATCC 33591S.aureus: wells 3: negative control of CC 25923 of S. aureus: wells (15-4): clinical sample

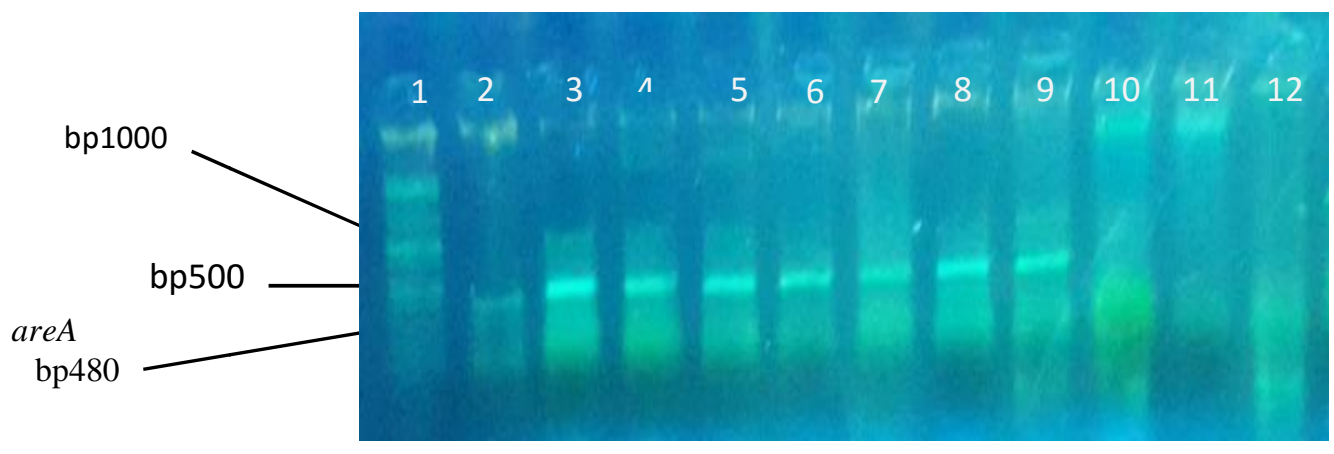

Fig.1. PCR reaction in $\operatorname{arcAgene}$ in isolates of MRSE: Well 1: bp 100 molecular weight marker, wells 2: negative control of ATCC 25923 of S.aureus: wells 3: positive control of CC 33591 of S. aureus: wells (15-4): clinical sample

\section{REFERENCES}

Akhtardanesh, L., Saeedi N., Z., Sarmadian, H. et al., 2015, to determine the nasal frequency and virulence of Staphylococcus isolated of nose of patients admitted in intensive care in Arak in, Journal Of Medical Sciences, 2014, 18(8), 1-10.

artels, Matte Damkjaer, Hansen, Hestbjerg Lars, And et al. (2011). An Unexpected location of the Arginine Catabolic Mobile element $(A C M E)$ in a USA300-Related MRSA Strain. Pols one. Vol. 6. No. 1. 1-8.

Brown, ML, et al. (2012). Prevalence and sequence variation of panton-valentine Leukocidin in the United State among methicillin-resistant and methicillin-susceptible Staphylococcus aureus. Journal of clinical microbiology. Vol. 50. No.1. 86-90. 
Borui, Pi, et al. (2009). Distribution of the $A C M E-\operatorname{arcA}$ gene among methicillin-resistant Staphylococcus haemolyticus and identification of a novel ccr allotype in ACME-arcAPositive isolates. Journal of medical microbiology. Vol. 58. No. 6. 731-736.

Barbier, F, et al. (2011). High prevalence of the arginine catabolic mobile element. In carriage isolates of methicillin-resistant Staphylococcus epidermidis. Journal Antimicrob Chemother. Vol. 66. No. 1. 29-36.

Du, Xin, et al. (2013). Mulecular Analysis of Staphylococcus epidermidis strains isolated from Community and Hospital Environment In Chaina. Plos One. Vol. 8. No. 5. 1-11

Diep, Binh An, et al. (2008). The arginie catabolic mobile element and Staphylococcal chromosomal cassette mec linkage: convergence of virulence and resistance in the USA300 clone of methicillin-resistant Staphylococcus aureus. Journal infectious diseases. Vol. 197. No.11. 1523-1530.

Degnan,B.A, et al. (2000). Characterization of an isogenic mutant of Streptococcus pyogenes manferdo laking the ability to make Streptococcal acid glycoprotein. Infect Immun. Vol. 68. 2441-2448.

Ellington, M. et al. (2008). Distribution of the ACME-arcA gene among methicillin-resistant Staphylococcus epidermidis from England and Wales. Journal of Antimicrobial chemotherapy. Vol. 61. 73-77.

Granslo, H. et al. (2010). Arginine Catabolic Mobile Element is associated With low antibiotic resistance and low pathogenicity in Staphylococcus epidermidis from neonates. Pediatric research. Vol. 68. 237-243.

Henegariu, O, Heerema, NA, Dlouhy, SR, Vance, GH, Vogt, PH. (1997). Multiplex PCR: and Critical parameters and Step-by- Step Porotocol. Biotechniqes. Vol. 23. 504-511.

Kondo, Y, Ito, T, Ma, XX, Watanabe, S, Kreiswirth, NB, Etienne, J, et al. (2007). Combination of Multiplex $\mathrm{PCR}_{\mathrm{s}}$ for Staphylococcal Cassette Choromosome mec Type Assignment: Rapid Idetification System for $m e c$, $c c r$, and Major Differences in Jounkyard Regious. Antimicrobial Agents and Chemotherapy, p: 264-274.

Kawaguchiya, M, et al. (2012). Characterization of PVL /ACME- Posetive Methicillin-resistant Staphylococcus aureus ( genotypes ST8-MRSA-IV and ST5-MRSA-II) isolated from a university hospital in Japan. Microb Drug Resist. Vol. 19. No. 1. 48-56. 
Kawaguchiya, M, et al. (2013). Genetic diversity of emerging Panton-Valentine Leukocidine / arginine catabolic mobile element (ACME)- positive ST8 SCCmec-IVa methicillin-resistant Staphylococcus aureus (MRSA) strains and ACME-positive CC5 (ST5 /ST764) MRSA strains in Northern Japan. Journal Medical Microbial. Vol. 62. No. 12. 1852-1863.

Lindgren, JK, Thomas, VC, Olson, ME, Chaudhari, SS, Nuxoll, AS, Schaeffer, CR, And et al. (2014). Arginine. Deiminase in Staphylococcus epidermidis Functions to Augments Biofilm Maturation through pH, Homeostasis. Journal of Bacteriology. Vol. 196. No. 12. 2277-2289. Miragaia, M., et al. (2009). Genetic diversity of Arginine catabolic mobile element. In Staphylococcus epidermidis. Pols one. Vol. 4. No. 11. E.7722.

Moncada, S, And Higgs, A. (1993). The L-arginine-nitric oxide pathway. Natural Engl Journal Medical. Vol. 329. 2002-2012.

Otto, M. (2011). Molecular basis of Staphylococcus epidermidis infection. Semin Immunopathol. Vol. 34. No.2. 201-214.

Onishi, M, et al. (2013). Prevalence and genetic diversity of Arginin Catabolic Mobile Element $(A C M E)$ in clinical isolates of coagulase-negative Staphylococci: identification of ACME Type I variants in Staphylococcus epidermidis. Infectious Genetic Evolation. Vol.20. 381-388.

Podbielski, A, et al. (1996). Molecular characterization of group A Streptococcal (GAS). Oligopeptide permease (opp) and its effect on cysteine protease production. Mol Microbial. Vol. 21. 1087-1099.

Palazzo, ICV, Araujo, MLC, Darini, A L C. (2005). First Report of Vancomycin-Resistant Staphylococci Isolated from Healthy Carriers in Brazil. Journal of Clinical Microbiology. Vol. 43. No.1.179-185.

Quinn, PJ, Carter, ME, Markey, BK, Carter, GR. (1994). Veterinary microbiology and microbial disease. International journal of current microbiology and applied sciences. Vol. 3. No. 2. 118-126.

Rosenthal, M, Dever, LL, Moucha, CS, Chavda, DK, Otto, M, and Kreiswrith, NB. (2011). Molecular. Characterization of an Early Invasive Staphylococcus epidermidis Prosthetic Joint infection. Microbial drug resistance. Vol. 17. No.3. 345-350. 
Sharma, V, Jindal, N, and Devi, P. (2010). Prevalence of methicillin resistant Coagulase negative. Staphylococci in a tertiary care hospital. Iranian Journal of Microbiology. Vol. 2. No.4. 185-188.

Shore, AC, et al. (2011). Characterization of a Novel Arginine Catabolic Mobile Element $(A C M E)$ and Staphylococcal. Chromosomal Cassette mec Composite Island with Significant Homology to Staphylococcus epidermidis. Type II in Methicillin-Resistant Staphylpcpccuus Aureus Genotype ST22-MRSA-IV. Antimicribial ACME Agents and Chemotherapy. Vol.55. No.5. 1896-1905.

Sabat, AJ, et al. (2015). Genome-wide analysis reveals two novel mosaic regions containing. An ACME with an identical DNA sequence in the MRSA ST398-t011 and MSSA ST8-t009 isolates. Journal Antimicrobe Chemother. Vol. 70. No. 5. 1298-1302.

Thurlow, Lance R, Joshi, Gauri S, and Richardson, Anthony R. (2012). Virulance strategies of the Dominant. USA300 Lineage of community associated methicillin resistant Staphylococcus aureus (CA-MRSA). FEMS Immunol Medical Microbial. Vol.65. No. 1. 5-22.

Urushibara,N, Kawaguchiya, M, And Kobayashi, N. (2012). Two novel arginine catabolic mobile elements and Staphylococcal chromosome cassette mec composite islands in community-acquired methicillin-resistant Staphylococcus aureus genotypes ST5-MRSA-V and ST5-MRSA-II. Journal Antimicrob Chemother. Vol. 67. No. 8. 1828-1834.

\section{How to cite this article:}

Shamansouri S, Zadeh VK, Khazaei M. A study on the percent of frequency of acme-arca in clinical isolates resistant to methicillin- staphylococcus epidermidis in Isfahan, Iran. J. Fundam. Appl. Sci., 2016, 8(2S), 1046-1057. 